\title{
Post-operative ocular infection due to Streptococcus dysgalactiae subspecies equisimilis
}

\author{
Jayaraman Kaliamurthy ${ }^{1}$, Vincenzo Cuteri ${ }^{2}$, Nelson Jesudasen ${ }^{1}$, Amjad Salman ${ }^{1}$, \\ Philip A Thomas ${ }^{1}$, Silvia Preziuso ${ }^{2}$ \\ ${ }^{1}$ Institute of Ophthalmology, Joseph Eye Hospital, Tiruchirapalli - 620001, Tamilnadu, India \\ ${ }^{2}$ Department of Veterinary Science, University of Camerino, Matelica (MC), Italy
}

\begin{abstract}
Ocular infections due to Streptococcus dysgalactiae subsp. equisimilis are rare. In the present report, three patients with a history of uncomplicated small incision cataract surgery with intraocular lens implantation developed exogenous endophthalmitis due to Streptococcus dysgalactiae subsp. equisimilis. The identification of the organisms was confirmed by PCR for a 16S rRNA sequence specific to the species $S$. dysgalactiae. Intravitreal treatment of cefazolin and amikacin, in addition to topical ofloxacin and tobramycin, resulted in resolution of infection in all three patients. Our reports indicate the importance of bacterial culture and molecular identification in the diagnosis of $S$. dysgalactiae subsp. equisimilis infection in the eye.
\end{abstract}

Key words: Streptococcus dysgalactiae subsp. Equisimilis; endophthalmitis; PCR; group G Streptococcus

J Infect Dev Ctries 2011; 5(10):742-744.

(Received 29 November 2010 - Accepted 16 March 2011)

Copyright $\odot 2011$ Kaliamurthy et al. This is an open-access article distributed under the Creative Commons Attribution License, which permits unrestricted use, distribution, and reproduction in any medium, provided the original work is properly cited.

\section{Introduction}

Streptococcus dysgalactiae subsp. equisimilis (group G beta-haemolytic streptococcus [GGS]) commonly inhabits the throat, skin, and vagina of healthy humans [1]. However, occasionally, the organism can cause pharyngitis, impetigo, cellulitis, septicemia, glomerulonephritis, and toxic shock [1]. The same disease spectrum generally coincides with that of a well-known human pathogen, the group A beta-haemolytic Streptococcus (GAS; Streptococcus pyogenes) which cohabits the same tissue sites. Due to the extensive overlap of the disease spectrum of GGS with GAS, it is possible that disease burden specifically attributed to GGS has been historically underestimated. Recent reports suggest an increase of GGS infections associated with classic human GAS-associated diseases, including invasive manifestations [2,3]. Phylogenetic analyses based on rRNA sequences and other molecular clocks have revealed that Streptococcus dysgalactiae spp. are closely related to $S$. pyogenes [4]. However, there have hitherto been only a few case reports describing ocular infection due to Streptococcus dysgalactiae subsp. equisimilis [5,6]. In the present report, ocular infection due to Streptococcus dysgalactiae subsp. equisimilis is described in three immunocompetent patients following ocular surgery.

\section{Case reports}

Three patients (a 50-year-old female, a 75-yearold male, and a 62-year-old male) all had a history of uncomplicated small incision cataract surgery with posterior chamber intraocular lens implantation in one eye. All three patients had been operated on the same day at the same surgical session in the same operating room and by the same surgeon. They did not receive immunosuppressive therapy, had no history of intravenous drug abuse, and were not alcoholics. There was no past history of diabetes mellitus or tuberculosis and no history of systemic infections on the day of surgery. All three patients developed pain and reduced vision in the operated eye within a week after surgery. At presentation, there were no signs and symptoms of infection in the unoperated eyes (pseudophakia in two patients). However, the operated eyes were inflamed with a dense peripheral corneal infiltrate and exudates in the anterior chamber and behind the intraocular lens. There was no view of the fundus and visual acuity was hand movements only. Ultrasound B scan revealed the presence of dense vitreous infiltrates with multiple cavities. The patients received intravitreal cefazolin $2.25 \mathrm{mg} / 0.1$ $\mathrm{ml}$ and amikacin $0.4 \mathrm{mg} / 0.1 \mathrm{ml}$, in addition to topical ofloxacin $(0.3 \%)$ and tobramycin $(0.3 \%)$. Vitreous material obtained from the inflamed eyes at the time of the intravitreal injection was 
subjected to detailed microbiological investigations. Following treatment, the corneal infiltrate decreased and exudates in the anterior chamber were also reduced. The patients were discharged on topical medications and asked to attend regular follow-up.

\section{Microbiological investigations}

Vitreous aspirates from the patients' infected eyes were processed aseptically for microbiological culture and direct microscopy. The samples were inoculated onto various culture media, including sheep blood agar, brain heart infusion agar and broth, and Sabouraud's dextrose agar and broth. The culture plates were incubated appropriately. Smears were prepared from the vitreous aspirates for Gram staining and also for lactophenol cotton blue mount. Microbiological surveillance of the operation room was performed to determine the possible source of the infection. For this purpose, throat swabs were obtained from the operation theatre personnel (two ophthalmologists, two surgical nurses, two floor nurses, and one assistant) who had been involved in the surgical procedures.

After microbiological investigations (culture), the organism was confirmed by PCR for a $401 \mathrm{bp}$ sequence of the 16S rRNA specific to the species $S$. dysgalactiae [7] and for a $601 \mathrm{bp}$ sequence of the streptokinase precursor gene specific to human isolates of Streptococcus dysgalactiae subspecies equisimilis [8] (H46skc-F: 5'GGTACTGTTGAGGGGACGAA-3' and H46skcR: 5'-CGATTGAGGAGTCACGTTCA-3'). The DNA was extracted from one loopful of a pure streptococcal culture by lysozyme/proteinase $\mathrm{K}$ digestion [9]. The PCR mixture contained $2 \mu \mathrm{l}$ of the template (microbial) DNA, 10 pmol of each primer, $25 \mu \mathrm{l}$ of Taq PCR mastermix (Qiagen $\mathrm{GmbH}$, Hilden, Germany) and water up to $50 \mu \mathrm{l}$ total volume. The PCR programme was as follows: $94^{\circ} \mathrm{C}$ for 3 minutes; 35 cycles at $94^{\circ} \mathrm{C}$ for 30 seconds, at $57^{\circ} \mathrm{C}$ for 30 seconds, and at $72^{\circ} \mathrm{C}$ for 40 seconds; followed by a final extension at $72^{\circ} \mathrm{C}$ for 7 minutes. Ten microliters of the reaction products were visualised by electrophoresis in $2.0 \%$ agarose gel containing $0.5 \mathrm{mg} / \mathrm{ml}$ of ethidium bromide with Tris-borate-EDTA buffer (TBE; $89 \mathrm{mM}$ Tris, $89 \mathrm{mM}$ boric acid, 2mM EDTA, $\mathrm{pH} 8.3$ ).

\section{Results and discussion}

Gram-stained smears of the vitreous fluid samples revealed numerous Gram-positive cocci in pairs and short chains; culture of these samples grew beta-haemolytic streptococci provisionally identified as Streptococcus dysgalactiae subsp. equisimilis by biochemical methods. Association of group G Streptococcus with endophthalmitis is well-established. Previously, such infection has been reported following cataract surgery, penetrating keratoplasty and trabeculectomy [10]. In addition, nine cases of endogenous Lancefield Group G beta-haemolytic Streptococcus (GGS)related endophthalmitis have been published so far. Of them, five cases were associated with endocarditis, one each with cellulitis of the foot, facial trauma, abscessed tooth and of unknown origin [6,11-13]. An additional study also indicates the involvement of this organism in bilateral endogenous endophthalmitis [14].

Due to the susceptibility pattern of the organism, various options are available to treat GGS related endoopthalmitis. In 1983, Ramasamy et al. [5] reported two cases of Streptococcus equisimilis ophthalmic infection, one with endophthalmitis and the other with purulent conjunctivitis; the endophthalmitis responded well to intravenous penicillin $\mathrm{G}$ and topical chloramphenicol while the purulent conjunctivitis resolved with Neosporin eye drops and vitamin supplements [5]. Recently, Suemori et al. [6] reported one case of Streptococcus equisimilis endogenous endophthalmitis whose general condition and ophthalmic inflammation improved following emergency mitral valvuloplasty, intravitreal and subconjunctival vancomycin and meropenem, topical levofloxacin and ofloxacin, and intravenous gentamicin and penicillin $\mathrm{G}$; however, the best-corrected visual acuity remained at light perception. In the present report, intravitreal administration of cefazolin and amikacin with the topical application of ofloxacin and tobramycin resulted in resolution of the infection in all the patients. The use of intravitreal antibiotics to treat GGS-related endogenous endophthalmitis is still controversial [11]. Further research on this aspect would facilitate successful treatment and outcome.

In this study, surveillance investigations showed the presence of Streptococcus dysgalactiae subsp. equisimilis in a throat swab sample collected from a 35-year-old healthy nurse who had assisted at the three surgeries. The nurse did not have a history of systemic infection and had no signs of upper respiratory tract infection or other diseases. The observation indicates the importance of infection control practices in hospital settings to avoid nosocomial infections; otherwise, the organisms may be transmitted from patients or the nursing staff or may originate from the hospital environment (exogenous infection). In the case of poor hygienic conditions, infection may be derived from the patient's own flora (endogenous infection). In either case, surgical procedures, 
instrumental manipulation, or nursing procedures play important roles in the spread of nosocomial infection. The situation can be worse in cases of surgical site infections that account for $14 \%-16 \%$ of all nosocomial infections [15]. In the present study, the surveillance results suggest that the nurse may have been a healthy carrier who directly transmitted the infection. The infection may also have been transmitted during surgery.

Epidemiology of Streptococcus dysgalactiae subsp. equisimilis is probably underestimated, and antibiotic treatment is often chosen by physicians without bacterial identification. However, due to its potential to spread in nosocomial settings, emergence of antibiotic resistance in these strains can be expected. Therefore, it is important to confirm the identification by bacterial culture and PCR and conduct an antibiotic susceptibility profile, especially for the strains isolated from the eye. Lab diagnostic procedures not only help to better investigate the spread and pathogenic potential of $S$. dysgalactiae subsp. Equisimilis but to evaluate the risk of nosocomial infections due to asymptomatic carriers.

\section{References}

1. Rolston KV (1986) Group G streptococcal infections. Arch Intern Med 146: 857-858.

2. Ekelund K, Skinhoj P, Madsen J, Konradsen HB (2005) Invasive group A, B, C and G streptococcal infections in Denmark 1999-2002: epidemiological and clinical aspects. Clin Microbiol Infect 11: 569-576.

3. Laupland KB, Ross T, Church DL, Gregson DB (2006) Population-based surveillance of invasive pyogenic streptococcal infection in a large Canadian region. Clin Microbiol Infect 12: 224-230.

4. Tapp J, Thollesson M, Herrmann B (2003) Phylogenetic relationships and genotyping of the genus Streptococcus by sequence determination of the RNase P RNA gene, rnpB. Int J Syst Evol Microbiol 53: 1861-187.

5. Ramaswamy G, Ambrose NG, Lockland Quinlan SM, Tchertkoff V (1983) Streptococcus equisimilis (Group C) as a cause of ophthalmic infections. Am J Clin Pathol 79: 385-387.

6. Suemori S, Sawada A, Komori S, Mochizuki K, Ohkusu K, Takemura K (2010) Case of endogenous endophthalmitis caused by Streptococcus equisimilis. Clin Ophthalmol 4: 917-918.

7. Riffon R, Sayasith K, Khalil H, Dubreuil P, Drolet M, Lagacé J (2001) Development of a rapid and sensitive test for identification of major pathogens in bovine mastitis by PCR. J Clin Microbiol 39: 2584-2589.

8. Caballero AR, Lottenberg R, Johnston KH (1999) Cloning, expression, sequence analysis, and characterization of streptokinase secreted by porcine and equine isolates of Streptococcus equisimilis. Infect Immun 67: 6478-6486.

9. Preziuso S, Laus F, Tejeda AR, Valente C, Cuteri V (2010) Detection of Streptococcus dysgalactiae subsp. equisimilis in equine nasopharyngeal swabs by PCR. J Vet Sci 11: 67-72.

10. Goh ES and Liew GC (2008) Traumatic blebassociated Group-G beta-haemolytic Streptococcus endophthalmitis. Clin Experiment Ophthalmol 36: 580-581.

11. Tan JH, Newman DK, Burton RL (1999) Endogenous endophthalmitis due to group $G$ streptococcus. Eye 13:116-117.

12. Ziakas NG, Tzetzi D, Boboridis K, Georgiadis NS (2004) Endogenous group G Streptococcus endophthalmitis following a dental procedure. Eur J Ophthalmol 14: 59-60.

13. Gopalamurugan $A B$, Wheatcroft $S$, Hunter $P$, Thomas $M R$ (2005) Bilateral endophthalmitis and ARDS complicating group G streptococcal endocarditis. Lancet 366: 2062.

14. Wickramasinghe $\mathrm{N}$ and Haris $\mathrm{K}$ (2010) Bilateral endophthalmitis as a primary manifestation of Streptococcus dysgalactiae endocarditis and the role of 16S rDNA polymerase chain reaction in identification. Diagn Microbiol Infect Dis 67: 185-187.

15. Emori TG, Gaynes RP (1993) An overview of nosocomial infections, including the role of the microbiology laboratory. Clin Microbiol Rev 6: 428442.

\section{Corresponding author}

Kaliamurthy $\mathbf{J}$

Department of Ocular Microbiology

Institute of Ophthalmology

Joseph Eye Hospital

Tiruchirapalli - 620001

Tamilnadu, India

Telephone: +91- 431 - 2460622, 2462862, 2414969

Fax: +91- $431-2414969$

Email: kaliaj17@gmail.com

Conflict of interests: No conflict of interests is declared. 